\title{
Modelling of the wave fields by the modification of the matrix method in anisotropic media
}

\section{A. Pavlova}

Carpatian Branch of Subbotin Institute of geophysics NAS of Ukraine, Lviv, Ukraine

Received: 17 December 2013 - Accepted: 7 January 2014 - Published: 3 February 2014

Correspondence to: A. Pavlova (susyinet@gmail.com)

Published by Copernicus Publications on behalf of the European Geosciences Union.

Modelling of the wave

fields by the

modification of the

matrix method

A. Pavlova

\section{Title Page}

\section{Abstract}

Conclusions

Tables

14

4

Back 


\section{Abstract}

The modification of the matrix method of construction of wave field on the free surface of an anisotropic medium is presented. The earthquake source represented by a randomly oriented force or a seismic moment tensor is placed on an arbitrary boundary 5 of a layered anisotropic medium. The theory of the matrix propagator in a homogeneous anisotropic medium by introducing a "wave propagator" is presented. It is shown that, for an anisotropic layered medium, the matrix propagator can be represented by a "wave propagator" in each layer. The matrix propagator $P\left(z, z_{0}=0\right)$ acts on the free surface of the layered medium and generates stress-displacement vector at depth $z$.

10 The displacement field on the free surface of an anisotropic medium is obtained from the received system of equations considering the radiation condition and that the free surface is stressless. The approbation of the modification of the matrix method for isotropic and anisotropic media with TI symmetry is done. A comparative analysis of our results with the synthetic seismic records obtained by other methods and published 15 in foreign papers is executed.

\section{Introduction}

The problem of wave fields modelling, when the source is presented by seismic moment, has practical applications in seismology. Therefore, the development of methods for determining the displacement field on the free surface of an anisotropic inhomogeneous medium for sources of this type is an actual task and needs to be resolved.

The propagation of seismic waves in inhomogeneous anisotropic medium is considered. A modification to the matrix method for constructing wavefield on the free surface of an anisotropic medium is presented. The earthquake source represented by a randomly oriented force or a seismic moment tensor is placed on an arbitrary boundary of a layered anisotropic medium. The theory of the matrix propagator in a homogeneous anisotropic medium by introducing a "wave propagator" is presented. It is shown that
SED

$6,467-485,2014$

\section{Modelling of the wave fields by the \\ modification of the matrix method \\ A. Pavlova}

Title Page
Abstract

Conclusions

Tables

14

4

Back
Introduction

References

Figures

$\rightarrow$

Close
Full Screen / Esc

Printer-friendly Version

Interactive Discussion 
for anisotropic layered medium the matrix propagator can be represented by a "wave propagator" in each layer. The displacement field on the free surface of an anisotropic medium is obtained from the received system of equations considering the radiation condition and that the free surface is stressless.

5 In recent years one of the most important methods is the development of approaches for constructing the theoretical seismograms, which allow the study of the structure of the medium and determination of the earthquake source parameters. The effects on the wave field and seismic waves' propagation in the earth's interior should be considered when calculating these seismograms. Thus, the displacement field, which 10 is registered on the free surface of an inhomogeneous medium, depends on the model of the geological structure and the physical processes in the source.

In the 1950s, Thomson and Haskell first proposed a method for constructing interference fields by simulation of elastic waves in layered isotropic half-space with planar boundaries (Haskell, 1953). The matrix method was developed in previous works 15 (Behrens, 1967; Buchen, 1996; Cerveny, 2001; Chapman, 1974).

The stable algorithms of seismogram calculation for all angles of seismic wave propagation are obtained. The matrix method is generalized for low-frequency waves in inhomogeneous elastic concentric cylindrical and spherical layers surrounded by an elastic medium. The concept of the characteristic matrix determined by physical parameters of the environment is developed. The matrix method is used for wave propagation in elastic, liquid and thermoelastic media. In addition, it has been generalized for the study of other processes described by linear equations. The advantage of the matrix method is the ability to write matrix expressions compactly that are useful both in analytical studies and numerical calculations.

The matrix method and its modifications are used to simulate the seismic wave propagation in isotropic and anisotropic media. This method is quite comfortable and has several advantages over other approaches. Both advantages and disadvantages of the matrix method are well described in Helbig et al. (2001), Stephen (1981), and Thomson (1950).

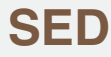

$6,467-485,2014$

\section{Modelling of the wave fields by the \\ modification of the matrix method \\ A. Pavlova}

\section{Title Page}


Today in seismology much attention is given to mathematical modelling as one of the main tools for the analysis and interpretation of the wave fields.

\section{Theory}

The problem of wave fields modelling, when the source is presented by seismic mo5 ment, has practical applications in seismology. Therefore, the development of methods for determining the displacement field on the free surface of an anisotropic inhomogeneous medium for sources of this type is an actual task and needs to be resolved.

In this paper the propagation of seismic waves in anisotropic inhomogeneous medium is modelled by a system of homogeneous anisotropic layers, as shown in

10 Fig. 1. Each layer is characterized by the propagation velocity of $P$ and $S$ wave and density. At the boundaries between layers, hard contact condition is met, except for the border, where the source of seismic waves is located.

The earthquake source is modelled by nine pairs of forces, which represented a seismic moment tensor. This description of the point source is sufficiently known and effec15 tive for simulation of seismic waves in layered half-space (Haskell, 1953). In general, the source is also assumed to be distributed over time (i.e. seismic moment $M_{0}(t)$ is a function of time). This means that the physical process in the source does not occur instantaneously, but within a certain time frame. It is known for our seismic events $(\mathrm{Mw} \sim 2-3)$ that the time during which the event occurred may be $0.1-0.7 \mathrm{~s}$. The determination of the source time function is an important seismic problem. In this chapter the direct problem solution is shown, when a point source is located on an arbitrary boundary of layered anisotropic media.

We assume the usual linear relationship between stress $\tau_{i j}$ and strain $e_{k l}$ :

$\tau_{i j}=c_{i j k l} \cdot e_{k l}=c_{i j k l} \frac{\partial u_{l}}{\partial x_{k}}$

where $\boldsymbol{u}=\left(u_{x}, u_{y}, u_{z}\right)^{\top}$ is displacement vector.

\section{SED}

$6,467-485,2014$

Modelling of the wave fields by the modification of the matrix method

A. Pavlova

Title Page

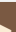

ose

C

\section{ion}


The equation of motion for an elastic homogeneous anisotropic medium, in the absence of body forces, is (Fryer et al., 1984)

$\rho \frac{\partial^{2} u_{i}}{\partial t^{2}}=c_{i j k l} \frac{\partial^{2} u_{l}}{\partial x_{i} \partial x_{k}}$,

5 where $\rho$ is the uniform mass density, and $c_{i j k l}$ are the elements of the uniform elastic coefficient tensor.

Taking the Fourier transform of Eqs. (1) and (2), we obtain the matrix equation (Fryer et al., 1987):

$\frac{\partial \boldsymbol{b}}{\partial z}=j \omega A(z) \boldsymbol{b}(z)$

where $\boldsymbol{b}=\left(\begin{array}{l}\boldsymbol{u} \\ \boldsymbol{\tau}\end{array}\right)$ is the vector of displacements and scaled traction, and $\boldsymbol{\tau}=$ $-\frac{1}{j \omega}\left(\tau_{x z}, \tau_{y z}, \tau_{z z}\right)^{\top}$. With the definition of $\boldsymbol{b}$ the system matrix $\mathbf{A}$ has the structure $\mathbf{A}=\left(\begin{array}{cc}\mathbf{T} & \mathbf{C} \\ \mathbf{S} & \mathbf{T}^{\boldsymbol{\top}}\end{array}\right)$; where $\mathbf{T}, \mathbf{S}$ and $\mathbf{C}$ are $3 \times 3$ submatrices, $\mathbf{C}$ and $\mathbf{S}$ are symmetric.

For any vertically stratified medium, the differential system Eq. (3) can be solved 15 subject to specified boundary conditions to obtain the response vector $\boldsymbol{b}$ at any desired depth. If the response at depth $z_{0}$ is $\boldsymbol{b}\left(z_{0}\right)$, the response at depth $z$ is

$\boldsymbol{b}(z)=P\left(z, z_{0}\right) \boldsymbol{b}\left(z_{0}\right)$,

where $P\left(z, z_{0}\right)$ is the stress-displacement propagator.

20 To find this propagator, it is necessary to find the eigenvalues (vertical slowness), the eigenvector matrix $\mathbf{D}$, and its inverse $\mathbf{D}^{-1}$ (Fryer et al., 1984):

$P\left(z, z_{1}\right)=\mathbf{D Q}\left(z, z_{1}\right) \mathbf{D}^{-1}$,
SED

$6,467-485,2014$

Modelling of the wave

fields by the

modification of the

matrix method

A. Pavlova

\section{Title Page}


where $\mathbf{Q}$ is the "wave" propagator (Fryer et al., 1984):

$\mathbf{Q}\left(z, z_{1}\right)=\left(\begin{array}{cc}E_{u} & 0 \\ 0 & E_{D}\end{array}\right)$

SED

where $E_{u}=\operatorname{diag}\left[e^{j \omega\left(z-z_{1}\right) q_{p}^{u}}, e^{j \omega\left(z-z_{1}\right) q_{s_{1}}^{u}}, e^{j \omega\left(z-z_{1}\right) q_{s_{2}}^{u}}\right]$,

$E_{\mathbf{D}}=\operatorname{diag}\left[e^{j \omega\left(z-z_{1}\right) q_{p}{ }^{\mathrm{D}}}, e_{s_{1}}^{j \omega\left(z-z_{1}\right) q^{\mathrm{D}}}, e_{s_{2}}^{j \omega\left(z-z_{1}\right) q^{\mathrm{D}}}\right]$.

Modelling of the wave

fields by the

modification of the

matrix method

tion of the propagator is straightforward. In the anisotropic case, analytic solutions have been found only for simple symmetries, so in general solutions will be found numerically.

The layered anisotropic medium, which consists of $n$ homogeneous anisotropic layers on an anisotropic half-space $(n+1)$ (Fig. 1), is considered. The source in the form of a jump in the displacement stress $\boldsymbol{F}=\boldsymbol{b}_{s+1}-\boldsymbol{b}_{\mathrm{s}}$ is placed on the $s$ boundary (Fig. 1); it is easy to write the following matrix equation, using Eqs. (13) and (14):

A. Pavlova

$\boldsymbol{b}_{n+1}=\left.P_{n, s} \boldsymbol{b}_{s+1}\right|_{z=z_{\mathrm{s}}}, v_{n+1}=\left.D_{n+1}^{-1} D_{n} Q_{n} D_{n}^{-1} \cdots D_{s+1} Q_{s+1} D_{s+1}^{-1} \cdot \boldsymbol{b}_{s+1}\right|_{z=z_{s}}$,

$\left.\boldsymbol{b}_{\mathrm{s}}\right|_{z=z_{\mathrm{s}}}=P_{s, s-1} P_{s-1, s-2} \cdots P_{2,1} P_{1,0} \cdot \boldsymbol{b}_{0}=D_{\mathrm{s}} Q_{\mathrm{s}} D_{\mathrm{s}}^{-1} \cdots D_{1} Q_{1} D_{1}^{-1} \cdot \boldsymbol{b}_{0}$,

$15 v_{n+1}=D_{n} Q_{n} D_{n}^{-1} \cdots D_{s+1} Q_{s+1} D_{s+1}^{-1} \cdot\left(b_{s}+F\right)=G^{n+1, s+1} \cdot\left(G_{s, 1} b_{0}+F\right)=$ $G^{n+1, s+1} G_{s, 1} \boldsymbol{b}_{0}+G^{n+1, s+1} \cdot \boldsymbol{F}=G \boldsymbol{b}_{0}+G^{n+1, s+1} \cdot \boldsymbol{F}$,

where

is a characteristic matrix of a layered anisotropic medium.

$\boldsymbol{v}_{n+1}=G \boldsymbol{b}_{0}+G \cdot G_{s, 1}^{-1} \cdot \boldsymbol{F}=G\left(\boldsymbol{b}_{0}+G_{s, 1}^{-1} \cdot \boldsymbol{F}\right)=G\left(\boldsymbol{b}_{0}+\tilde{\boldsymbol{F}}\right)$,

Title Page

Abstract

Introduction

Conclusions

References

Tables

Figures

14

4

Back

Close

Full Screen / Esc

Printer-friendly Version

Interactive Discussion 
where $\tilde{F}=G_{s, 1}^{-1} \cdot F, G=G^{n+1, s+1} \cdot G_{s, 1}$. Using Eq. (7) and the radiation condition (with a halfspace $(n+1)$ the waves are not returned), and also the fact that the tension on the free surface equals to zero, we obtain a system of equations:

$6,467-485,2014$

$\left(\begin{array}{c}0 \\ 0 \\ 0 \\ v_{D}^{P} \\ s_{D} \\ v_{D} \\ v_{D}\end{array}\right)=\left(\begin{array}{llllll}G_{11} & G_{12} & G_{13} & G_{14} & G_{15} & G_{16} \\ G_{21} & G_{22} & G_{23} & G_{24} & G_{25} & G_{26} \\ G_{31} & G_{32} & G_{33} & G_{34} & G_{35} & G_{36} \\ G_{41} & G_{42} & G_{43} & G_{44} & G_{45} & G_{46} \\ G_{51} & G_{52} & G_{53} & G_{54} & G_{55} & G_{56} \\ G_{61} & G_{62} & G_{63} & G_{64} & G_{65} & G_{66}\end{array}\right)\left(\begin{array}{c}u_{x}^{(0)}+\tilde{F}_{1} \\ u_{y}^{(0)}+\tilde{F}_{2} \\ u_{z}^{(0)}+\tilde{F}_{3} \\ \tilde{F}_{4} \\ \tilde{F}_{5} \\ \tilde{F}_{6}\end{array}\right)$.

Modelling of the wave fields by the modification of the matrix method

A. Pavlova

Using only the homogeneous equations is sufficient to get the displacement field on a free surface:

$$
\left\{\begin{array}{l}
G_{11} u_{x}^{(0)}+G_{12} u_{y}^{(0)}+G_{13} u_{z}^{(0)}=-\left(G_{11} \tilde{F}_{1}+G_{12} \tilde{F}_{2}+G_{13} \tilde{F}_{3}+G_{14} \tilde{F}_{4}+G_{15} \tilde{F}_{5}+G_{16} \tilde{F}_{6}\right) \\
G_{21} u_{x}^{(0)}+G_{22} u_{y}^{(0)}+G_{23} u_{z}^{(0)}=-\left(G_{21} \tilde{F}_{1}+G_{22} \tilde{F}_{2}+G_{23} \tilde{F}_{3}+G_{24} \tilde{F}_{4}+G_{25} \tilde{F}_{5}+G_{26} \tilde{F}_{6}\right) \\
G_{31} u_{x}^{(0)}+G_{32} u_{y}^{(0)}+G_{33} u_{z}^{(0)}=-\left(G_{31} \tilde{F}_{1}+G_{32} \tilde{F}_{2}+G_{33} \tilde{F}_{3}+G_{34} \tilde{F}_{4}+G_{35} \tilde{F}_{5}+G_{36} \tilde{F}_{6}\right)
\end{array} .\right.
$$

10 The stress-displacement discontinuity is determined thought the components of the seismic tensor in matrix form (Fryer et al., 1984):

$$
F=\left(\begin{array}{l}
-c_{55}^{-1} M_{x z} \\
-c_{44}^{-1} M_{y z} \\
-c_{33}^{-1} M_{z z} \\
p_{x}\left(M_{x x}-c_{13} c_{33}^{-1} M_{z z}\right)+p_{y} M_{x y} \\
p_{x} M_{y x}+p_{y}\left(M_{y y}-c_{23} c_{33}^{-1} M_{z z}\right) \\
p_{x}\left(M_{z x}-M_{x z}\right)+p_{y}\left(M_{z y}-M_{y z}\right)
\end{array}\right) \delta\left(z-z_{z}\right),
$$


where $M_{x x}, M_{y y}, M_{z z}, M_{x z}, M_{y z}, M_{y x}, M_{x y}, M_{z y}$, and $M_{z x}$ are components of the seismic moment tensor, and $c_{13}, c_{23}, c_{33}, c_{44}$, and $c_{55}$ are components of the stiffness matrix.

As a result, the displacement field of the free surface of an anisotropic medium is in 5 the spectral domain as

$\boldsymbol{u}=\left(\begin{array}{c}u_{x}^{0} \\ u_{y}^{0} \\ u_{z}^{0}\end{array}\right)=\left(G^{13}\right)^{-1} \cdot \boldsymbol{y}$,

where

$G^{13}=\left(\begin{array}{lll}G_{11} & G_{12} & G_{13} \\ G_{21} & G_{22} & G_{23} \\ G_{31} & G_{32} & G_{33}\end{array}\right), \quad \boldsymbol{y}=\left(\begin{array}{l}a \\ b \\ c\end{array}\right)$

$10 \quad a=-\left(G_{11} \tilde{F}_{1}+G_{12} \tilde{F}_{2}+G_{13} \tilde{F}_{3}+G_{14} \tilde{F}_{4}+G_{15} \tilde{F}_{5}+G_{16} \tilde{F}_{6}\right)$,

$b=-\left(G_{21} \tilde{F}_{1}+G_{22} \tilde{F}_{2}+G_{23} \tilde{F}_{3}+G_{24} \tilde{F}_{4}+G_{25} \tilde{F}_{5}+G_{26} \tilde{F}_{6}\right)$,

$c=-\left(G_{31} \tilde{F}_{1}+G_{32} \tilde{F}_{2}+G_{33} \tilde{F}_{3}+G_{34} \tilde{F}_{4}+G_{35} \tilde{F}_{5}+G_{36} \tilde{F}_{6}\right)$.

Using Eq. (8) and three-dimensional Fourier transform, we obtain a direct problem time domain as

$\boldsymbol{u}\left(x, y, z_{R}, t\right)=\frac{1}{8 \pi^{3}} \iiint_{-\infty} \omega^{2} \boldsymbol{u}\left(p_{x}, p_{y}, z_{R}, \omega\right) e^{j \omega\left(t-p_{x} x-p_{y} y\right)} \mathrm{d} p_{x} \mathrm{~d} p_{y} \mathrm{~d} \omega$,

where $z_{R}$ is the epicentral distance and $p_{x}, p_{y}$ the horizontal slowness.

SED

$6,467-485,2014$

Modelling of the wave fields by the modification of the matrix method

A. Pavlova

Title Page

\section{Full Screen / Esc}

Printer-friendly Version 


\section{The comparative analysis}

A comparative analysis of synthetic seismograms is done to confirm the reliability of the described modification of the matrix method.

To test the proposed methodology used, results were obtained from the group of sci5 entists involved in the project Source Inversion Validation (SIV) (http://equake-rc.info/ sivdb/wiki/): Mathieu Causse (France), Susana Custodio (USA), Martin Mai (Saudi Arabia), Kaeser Martin (Germany), Haruko Sekiguchi (Japan), Guangfu Shao (USA), and Seok-Goo Song (Switzerland). The synthetic seismograms are calculated for known model of medium (Table 1) and locations of seismic stations (Fig. 2) by different methods: Kennett propagator matrix technique (A1, A2), Thomson-Haskell propagator matrix technique (ZR1, ZR2), finite element method (CS1, CS2, CS3), and finite-element method combined with the explicit time integration method using arbitrary high-order derivatives (DG). Figure 4 shows the synthetic seismograms (project SIV), as well as through the proposed modification of the matrix method, for medium modelled by five homogeneous layers. The source is located at a depth of $10 \mathrm{~km}$ (third layer), focal mechanism is a pure shear (Fig. 3), and the source time function is boxcar with rise time of $0.2 \mathrm{~s}$. Seismic moment is given as $M_{0}=3.4992 \times 10^{16} \mathrm{Nm}(\mathrm{Mw}=4996)$.

Focal mechanism, which is shown in Fig. 3, corresponds to the seismic moment tensor; all components are equal to zero except for $M_{x y}=M_{y x}$.

20 Comparative analysis of synthetic seismograms shows that the proposed modification of the matrix method for the determination of the displacement field on the free surface of the layered half-space can be used for modelling of wave fields.

In the paper by Chen (2008), numerical simulations of seismic wave propagation are presented. The author considers anisotropic and heterogeneous earth models, in particular, transversely isotropic PREM (The Preliminary Reference Earth Model) (Dziewonski et al., 1981) and weakly anisotropic model (isotropic PREM with $5 \%$ share of anisotropic perturbations). In this paper the synthetic seismograms are calculated by numerical method. For body-wave simulations, the source is replaced by a vertical

\section{SED}

$6,467-485,2014$

\section{Modelling of the wave fields by the \\ modification of the matrix method \\ A. Pavlova}

\section{Title Page}


point force located at a depth of $600 \mathrm{~km}$. The station is located at a source azimuth of $0^{\circ}$ (i.e. along the prime meridian) at an epicentral distance of $60^{\circ}$. The vertical point force avoids waveform complexities associated with the radiation pattern. The source-time function (STF) is a Ricker wavelet (17) with a $3 \mathrm{~s}$ half-duration:

$5 \quad \operatorname{STF}=\left(1-2 \pi^{2} f^{2} t^{2}\right) e^{-(\pi f t)^{2}}$.

Both seismograms are aligned on the $P$ arrival predicted by IASP91 (Kennett et al., 1991 ), and the IASP91 arrivals times of $P, \mathrm{PcP}, \mathrm{pP}, \mathrm{sP}, S$, and ScS are indicated by the arrows (Chen, 2008).

10 The waveforms computed by the proposed modification of the matrix method are very similar to the synthetic seismograms done in the paper (Chen, 2008).

\section{Conclusion}

In this paper the analytical methods for calculation of the displacement field on the free surface of a layered anisotropic medium (with transversely isotropic, orthorhombic and 15 monoclinic symmetry) are developed, when the source of seismic waves is presented by a randomly oriented force and/or seismic moment tensor. The stress-displacement discontinuity is determined via seismic moment tensor components. It is shown that the advantage of the matrix method is the possibility of compact solution recording of direct and inverse problems of seismology. The method of wave fields modelling in layered medium using eigenvectors and eigenvalues is developed. The method for determining the displacement field on the free surface of an anisotropic inhomogeneous medium from a source presented by a seismic moment tensor and a randomly oriented force is tested.

Probability and reliability of basic scientific terms and results is provided by wellposed problems, rigidity of mathematical methods and transformations in obtaining basic analytical relations for the displacement field and the seismic moment tensor
SED

$6,467-485,2014$

Modelling of the wave fields by the modification of the matrix method

A. Pavlova

Title Page

Abstract Introduction

Conclusions

References

Tables

Figures

I4

$\Delta$

4

Back

Close

Full Screen / Esc

Printer-friendly Version

Interactive Discussion 
components, by conducting computational experiments with reasonable accuracy, controlled by means of the theoretical relations for variations of physical parameters of studied media and wave forms on the surface of a layered half-space, and is also confirmed by the coincidence with analytical solutions and with results obtained by other 5 methods.

The approbation of the proposed method is done via comparative analysis of waveforms, obtained by different methods. Comparative analysis of waveforms confirms the possibility of using the matrix method for problems of seismology in the case of distributed in time earthquake sources for isotropic and anisotropic media. This paper is the first step in determining the earthquake source parameters. The algorithm to determine the source parameters is based on the expressions for displacement field on free surface of an anisotropic medium in Eq. (8) and spectra of real records from stations that recorded these events. The results of determining of the earthquake source parameters will be published in the next papers.

15 Thus, the methods, approaches, algorithms, software for the propagation of seismic waves and results of direct and inverse dynamic problems of seismology proposed and developed by the author and highlighted in the paper, can be successfully used in the study of the seismic regions and effective implementation in the construction of the earthquake source mechanism which is crucial for seismic regions of the country.

Probability and reliability of basic scientific terms and results is provided by well posed problems, rigidity of mathematical methods and transformations in obtaining basic analytical relations for the displacement field and the seismic moment tensor components, by conducting computational experiments with reasonable accuracy, controlled by means of the theoretical relations for variations of physical parameters of studied media and wave forms on the surface of a layered half-space, and is also confirmed by the coincidence with analytical solutions and with results obtained by other methods.

\section{SED}

$6,467-485,2014$

\section{Modelling of the wave fields by the modification of the matrix method \\ A. Pavlova}

\section{Title Page}




\section{References}

Behrens, E.: Sound propagation in lamellar composite materials and averaged elastic constants, J. Acoust. Soc. Am., 42, 168-191, 1967.

Buchen, P. W.: Ben-Hador R, Free-mode surface-wave computations, Geophys. J. Int., 124, 869-887, 1996.

Cerveny, V.: Seismic Ray Theory, Cambridge University Press, 2001.

Chapman, C. H.: The turning point of elastodynamic waves, Geophys. J. R. Astr. S., 39, 613621, 1974.

Chen, M.: Numerical simulations of seismic wave propagation in anisotropic and heterogeneous Earth models - the Japan subduction zone, California Institute of Technology, 143, 2008.

Crampin, S.: A review of the effects of anisotropic layering on the propagation of seismic waves, Geophys. J. R. Astr. S., 49, 9-21, 1977.

Crampin, S.: A review of wave motion in anisotropic and cracked elastic media, Wave Motion, 3, 343-391, 1981.

Dziewonski, A. M. and Andersin, D. L.: Preliminary reference Earth model, Phys. Earth Planet. In., 25, 297-356, 1981.

Fryer, G. J. and Frazer, L. N.: Seismic waves in stratified anisotropic media, Geophys. J. Roy. Astr. S., 78, 691-710, 1984.

20 Fryer, G. J. and Frazer, L. N.: Seismic waves in stratified anisotropic media - II. Elastodynamic eigensolutions for some anisotropic systems, Geophys J. Roy. Astr. S., 91, 73-101, 1987.

Haskell, N. A.: The dispersion of waves in multilayered media, B. Seismol. Soc. Am., 43, 17-34, 1953.

Helbig, K. and Treitel, S.: Wave Fields in Real Media: Wave Proragation in Anisotropic, Anelastic and Porous Media, Oxford, 2001.

Keith, C. M. and Crampin, S.: Seismic body waves in anisotropic media: propagation through a layer, Geophys. J. R. Astr. S., 49, 200-223, 1977a.

Kennett, B. L. N. and Engdahl, E. R.: Traveltimes for global earthquake location and phase identification, Geophys. J. Int., 105, 429-465, 1991.

30 Stephen, R. A.: Seismic anisotropy observed in upper oceanic crust, Geophys. Res. Lett., 8, 865-868, 1981.
SED

6, 467-485, 2014

Modelling of the wave fields by the

modification of the matrix method

A. Pavlova

Title Page

Abstract

Introduction

Conclusions

References

Tables

Figures

14

$\rightarrow 1$

Back

Close

Full Screen / Esc

Printer-friendly Version

Interactive Discussion 
Thomson, W. T.: Transmission of elastic waves through a stratified solid medium, J. Appl. Phys., 21, 89-93, 1950.
SED

$6,467-485,2014$

Modelling of the wave fields by the modification of the matrix method

A. Pavlova

Title Page

Abstract

Introduction

Conclusions

References

Tables

Figures

14

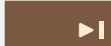

4

Back

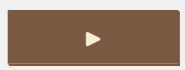

Close

Full Screen / Esc

Printer-friendly Version

Interactive Discussion 
$6,467-485,2014$

Modelling of the wave fields by the modification of the matrix method
A. Pavlova

Table 1. The parameters of medium.

\begin{tabular}{llllll}
\hline No. & layer thickness, $\mathrm{m}$ & $c_{11}, \mathrm{GPa}$ & $c_{12}, \mathrm{GPa}$ & $c_{44}, \mathrm{GPa}$ & density, $\mathrm{kg} \mathrm{m}^{-3}$ \\
\hline 1 & 2000 & 52992 & 21896 & 15548 & 2300 \\
2 & 2800 & 75625 & 27575 & 24025 & 2500 \\
3 & 13200 & 103788 & 33804 & 34992 & 2700 \\
4 & 6000 & 129472 & 48608 & 40432 & 2800 \\
5 & 21000 & 204800 & 68196 & 68302 & 3200 \\
\hline
\end{tabular}

Title Page

Abstract

Conclusions

Tables

14

4

Back

Full Screen / Esc

Printer-friendly Version

Interactive Discussion 


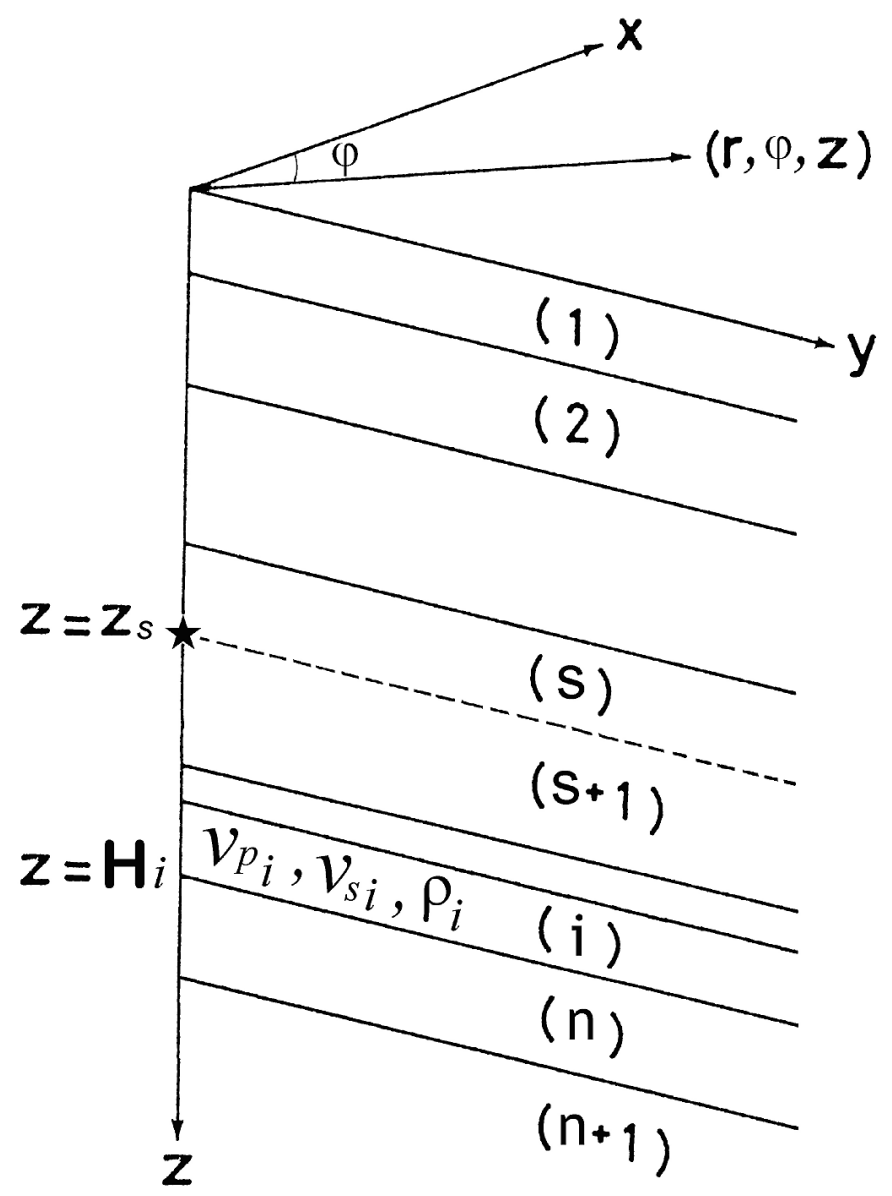

SED

$6,467-485,2014$

Modelling of the wave fields by the modification of the matrix method
A. Pavlova

Title Page

Abstract

Introduction

Conclusions

References

Tables

Figures

14

4

Back

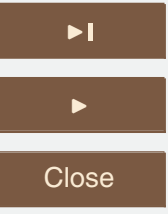

Full Screen / Esc

Printer-friendly Version

Fig. 1. Model vertically inhomogeneous medium.

Interactive Discussion 


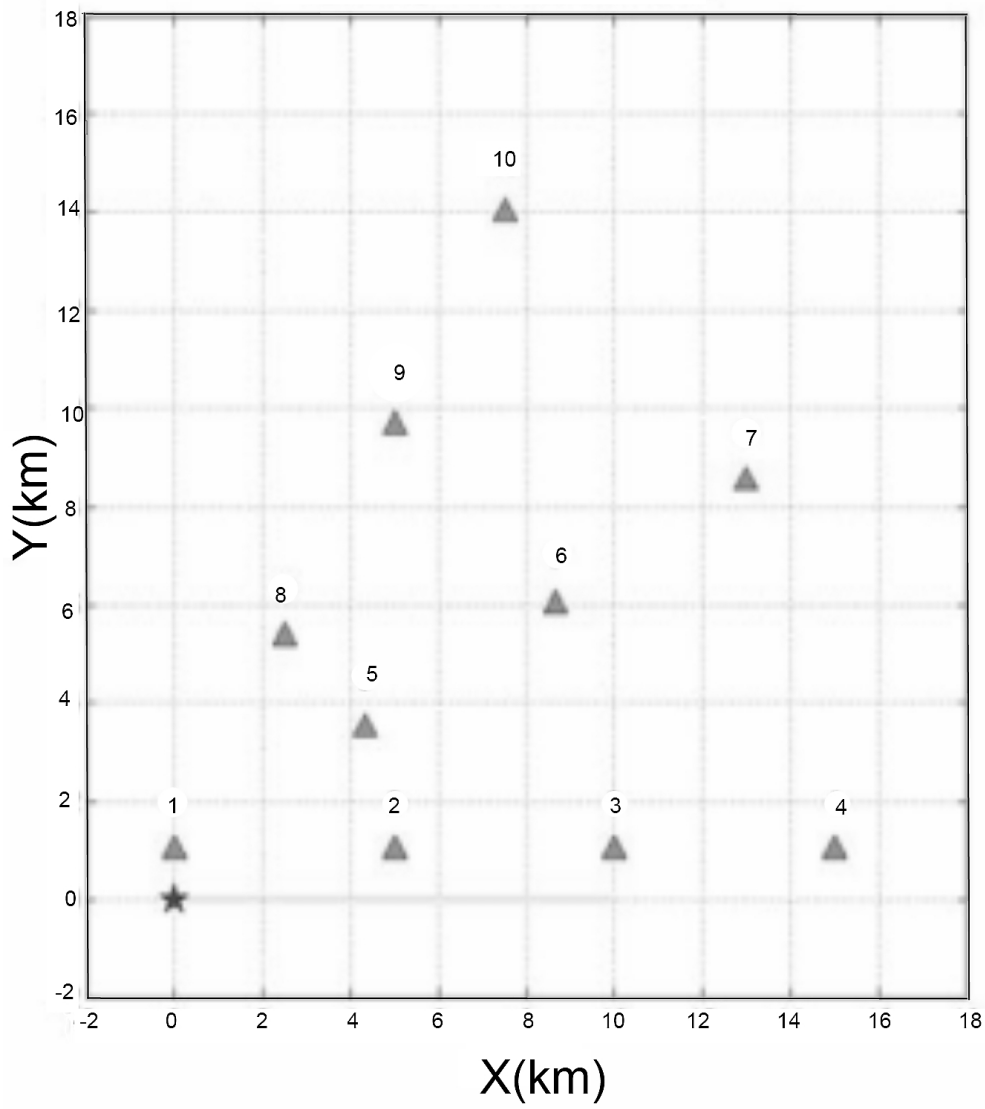

Fig. 2. Source-receiver geometry for the strike-slip point source. The star shows the epicentre in the chosen right-handed coordinate system with positive $X$ pointing east, positive $Y$ pointing north, and positive $Z$ pointing up.

\section{SED}

$6,467-485,2014$

Modelling of the wave fields by the modification of the matrix method
A. Pavlova

\section{Title Page}

Abstract

Conclusions
References

Tables

Figures

14

4

Back

Full Screen / Esc

Printer-friendly Version

Interactive Discussion 
$P$ axis $(\phi / \delta)$ in degrees: $135.00 / 0.00$

$T$ axis $(\phi / \delta)$ in degrees: $45.00 / 0.00$

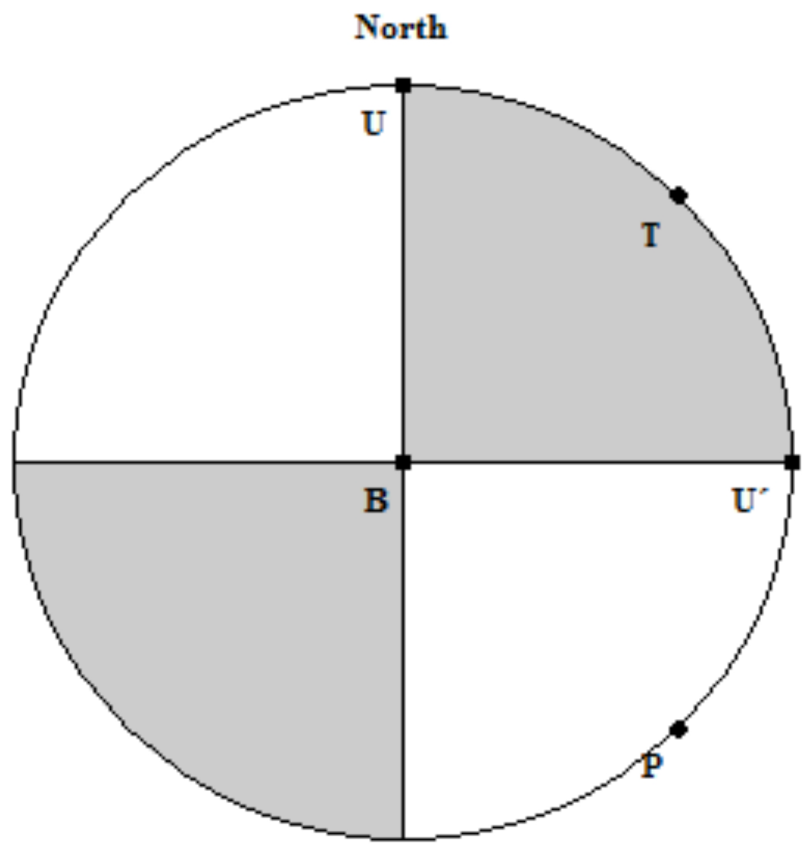

stereographic Aower

fault plane $(\phi / \delta / \lambda)$ in degrees: $0.00 / 90.00 / 0.00$

auxiliary plane $(\phi / \delta / \lambda)$ in degrees: $270.00 / 90.00 / 180.00$

Fig. 3. The source focal mechanism (strike-slip).

SED

$6,467-485,2014$

Modelling of the wave fields by the modification of the matrix method

A. Pavlova

\section{Title Page}

\section{Abstract}

Conclusions

Tables

14

Back

Full Screen / Esc

Printer-friendly Version

Interactive Discussion 
SED

$6,467-485,2014$
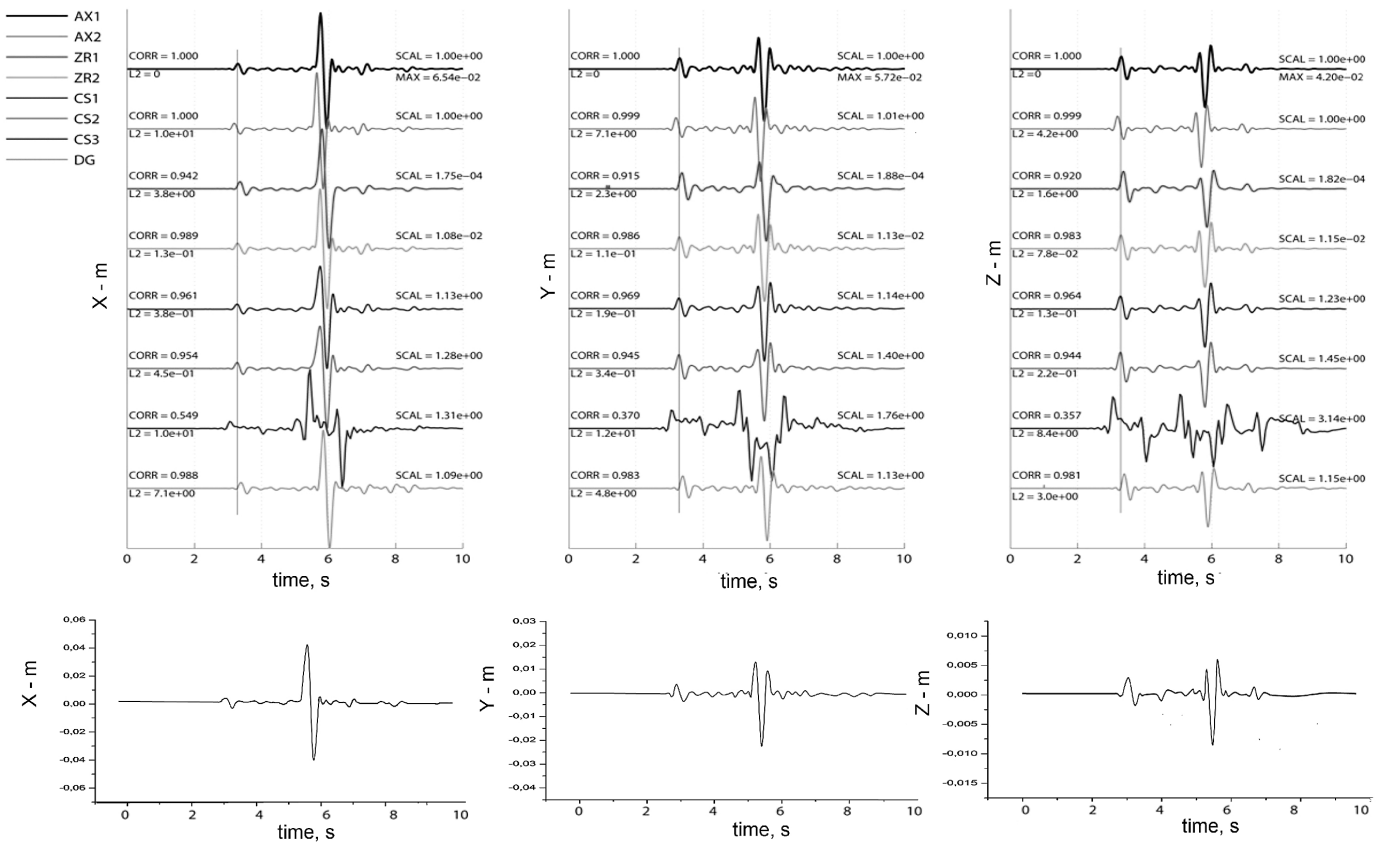

Fig. 4. Components of the displacement field on the free surface of the medium (Table 1), calculated by different methods according to the project SIV and by proposed modification of the matrix method for the receiver $10(X=13990 \mathrm{~m}, Y=7500 \mathrm{~m})$.
Modelling of the wave fields by the modification of the matrix method
A. Pavlova

\section{Title Page}

\section{Full Screen / Esc}

Printer-friendly Version

Interactive Discussion 
SED

$6,467-485,2014$

Modelling of the wave fields by the modification of the matrix method
A. Pavlova

a

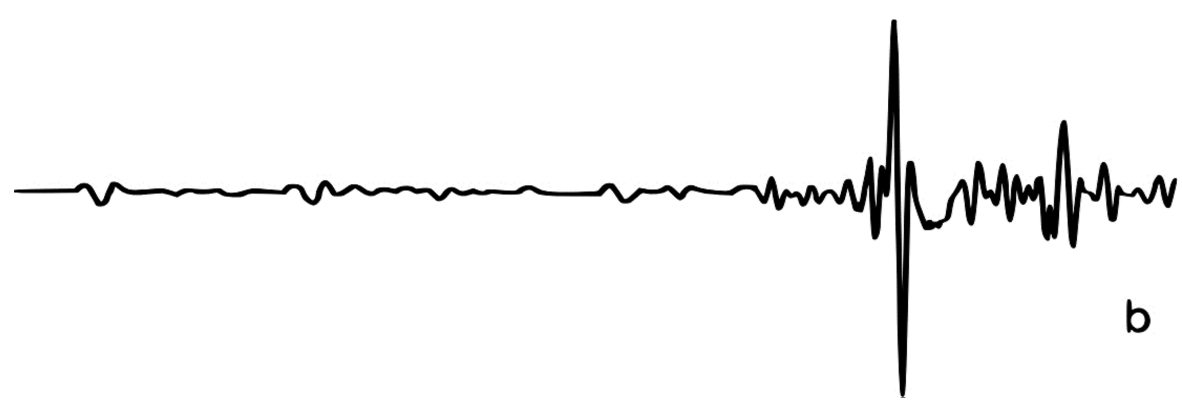

Fig. 5. (a) Synthetic seismograms for PREM and an anisotropic model (isotropic model with $5 \%$ share of weakly anisotropic perturbations). (b) Synthetic seismogram calculated by the proposed modification of the matrix method.

\section{Title Page}

Abstract

Conclusions

Tables

14

4

Back

Full Screen / Esc

Printer-friendly Version

Interactive Discussion 